\title{
Energy-Optimal Path Planning for Solar-Powered Aircraft in Level Flight
}

\author{
Andrew T. Klesh*and Pierre T. Kabamba ${ }^{\dagger}$ \\ University of Michigan, Ann Arbor, Michigan, 48109
}

\begin{abstract}
[Abstract] Motivated by long-endurance requirements in surveillance, reconnaissance and exploration, this paper considers level flight path planning for unmanned aerial vehicles (UAVs) equipped with solar cells on the upper surface of the wings. These solar cells collect energy that is stored in a battery and used to drive a propeller. The mission of the UAV is to travel from a given initial position to a given final position, in less than an allowed duration, using energy from the battery. The subsequent problem of energy-optimal path planning features the interaction between the aircraft kinematics, the energy collection model, and the energy loss model. All three models are coupled by the bank angle of the UAV. Within this framework, the problem is formulated as an optimal path planning problem, with the aircraft bank angle and speed serving as control inputs. Necessary conditions for optimality are given, whose solution yields extremal paths. These necessary conditions are utilized to study analytically the properties of extremal paths. An efficient numerical procedure is also given. It is shown that optimal paths belong to one of two regimes: solar or drag. The Power Ratio, a non-dimensional number that can be computed before flight, is identified. It is shown that this ratio predicts the regime of the optimal path, which facilitates the solution. Implications of the power ratio for UAV design are discussed. Several illustrations are given.
\end{abstract}

\section{Nomenclature}

$\begin{array}{llll}a & \text { Azimuth of the sun, deg } & K & \begin{array}{l}\text { Amount whereby the induced drag exceeds } \\ \text { that of an elliptical lift distribution }\end{array} \\ C_{D} & \text { Coefficient of drag } & & \text { X-Costate, N } \\ C_{D_{o}} & \text { Parasitic drag coefficient } & \lambda_{x} & \text {-Costate, N } \\ C l & \text { Coefficient of lift } & \lambda_{y} & \text { Y-Cost, } \mathrm{J} \\ D & \text { Drag of the aircraft, N } & \lambda_{\psi} & \Psi \text {-Costate, } \\ \delta & \text { Variation } & \phi & \text { Bank angle, deg } \\ \epsilon & \text { Oswald efficiency factor } & P_{\text {in }} & \text { Power collected, W } \\ e & \text { Elevation of the sun, deg } & P_{\text {out }} & \text { Power lost, W } \\ E_{\text {in }} & \text { Energy collected, J } & P_{R} & \text { Power Ratio } \\ E_{\text {out }} & \text { Energy lost, J } & P_{s d} & \text { Power spectral density of the sun, } W / m^{2} \\ E_{R} & \text { Energy Ratio } & \psi & \text { Heading, deg } \\ E_{T} & \text { Total Energy, J } & \rho & \text { Air density, } k g / m^{3} \\ \eta_{p r o p} & \text { Efficiency of the propeller } & S & \text { Surface area of the wing, } m^{2} \\ \eta_{s o l} & \text { Efficiency of the solar cells } & T & \text { Thrust of the aircraft, N } \\ g & \text { Gravitational acceleration, } m / s^{2} & t_{f} & \text { Final time, sec } \\ H & \text { Hamiltonian, W } & T_{M} & \text { Maximum time allowed, sec } \\ H_{\phi \phi} & \partial^{2} H / \partial \phi^{2} & t_{o} & \text { Initial time, sec }\end{array}$

*Graduate Student, Aerospace Engineering, aklesh@umich.edu

${ }^{\dagger}$ Professor, Aerospace Engineering, kabamba@umich.edu 


$\begin{array}{llll}H_{\phi V} & \partial^{2} H / \partial \phi \partial V & V & \text { Speed, m/s } \\ H_{V V} & \partial^{2} H / \partial V^{2} & x & \text { X position, m } \\ i & \text { Incidence angle of sun rays, deg } & W & \text { Weight of the aircraft, N } \\ j & \text { Dummy summation index } & y & \text { Y position, } \mathrm{m}\end{array}$

\section{Introduction}

Increasing endurance requirements within surveillance, reconnaissance and exploration missions require a new class of unmanned aerial vehicles (UAVs). The UAV considered in this paper is distinguished from the majority of UAVs by its power source. The aircraft discussed are equipped with solar cells on the upper surface of the wings as well as onboard energy storage. These solar cells collect energy that is used to drive a propeller.

This paper considers the problem of energy-optimal path planning for solar-powered UAVs in level flight. This problem features the interaction between three subsystems: aircraft kinematics, energy collection, and energy loss. While the current literature discusses methods to optimize UAV aerodynamic design for energy usage, there is no approach that examines the coupling of energy collection and energy loss with the aircraft kinematics. The purpose of this paper is to investigate this relationship and account for it in optimal path planning.

Although the current literature on solar-powered UAVs does not consider optimal energy path planning, a substantial body of work is available on the design and analysis of solar-powered aircraft. A brief review of this literature is as follows. The feasibility of solar-powered flight is reviewed in Refs. 1-2 with a reference to Dr. A. Raspet's pioneering proposal of solar-powered flight in 1954. Hence, solar-powered aircraft have only appeared recently and their history is discussed in Refs. 6, 9, and 26. The general history and methods for design and analysis of solar-powered aircraft are discussed in Refs. 3-27. References 12, 15 and 25 are unique in that they use an optimization procedure to design the aircraft based upon expected maneuvers and sunlight availability.

Optimal path planning for solar-powered aircraft is qualitatively discussed in the literature. Mission design is found in Refs. 27-30 with particular emphasis on where and when to fly. In most references, efficiency through preliminary design is emphasized. Alternative methods to increase efficiency for solarpowered aircraft are discussed in Refs. 43-45. Reference 45 is of particular importance as it achieves a $30 \%$ increase in efficiency by improving the cooling of solar cells. However, nowhere in the literature is there a study directed towards optimizing the flight path itself based upon the interaction of kinematics and energetics.

Solar-powered aircraft have many potential uses in exploration and civilian applications. References 31-35 propose innovative designs for the use of solar powered aircraft on Mars and Venus. In Refs. 36-41, additional proposals are made for high altitude wireless communication platforms and other uses.

The work in Ref. 31 does take into consideration optimal path planning when designing the flight of Helios. The maneuvers considered include takeoff, cruise and climb. An important note is that Ref. 31 considers winds in path planning. It does not, however, give a general analytical solution to the path planning problem. Moreover, it does not account for coupling between the turn rate of the aircraft and the incidence angle of the sun rays through aircraft bank angle.

The present paper presents an integrated model of the aircraft kinematics and energetics that has the following original features. First, the energy collected and lost depend upon the bank angle of the aircraft. The turn rate of the aircraft is also dependent upon the bank angle. Thus the aircraft kinematics and energetics are coupled through the bank angle. Second, the sun is not assumed to always be present in the sky. Maneuvers in all light conditions are considered and several regimes of flight are discussed.

Based on the integrated model, the problem of solar-powered UAV level flight path planning is formulated as a maximization optimal path-planning problem, with the bank angle and speed serving as control inputs. The present paper studies this optimization problem and provides the following original contributions:

- The necessary conditions for optimality for the maximization problem are formulated.

- From these necessary conditions, the properties of energy-optimal paths are derived.

- An efficient numerical optimization procedure is given. 
- Two distinct regimes of optimal flight are identified.

- Finally, the power ratio is shown to correctly predict the regime of optimal flight.

The remainder of the paper is as follows. In Sec. II, the model is presented. In Sec. III, the optimization problem is formulated for maximization of the total final energy of the UAV. In Sec. IV, the necessary conditions of optimality are used to characterize the optimal paths. Section V presents the discretization procedure used to numerically compute optimal paths, whereas in Sec. VI, the power ratio is introduced. In Sec. VII, properties of the optimal paths are presented. Section VIII discusses implications of the power ratio on preliminary design. Section IX provides conclusions and discusses future work. Derivation of the solar incidence angle, the properties of the aircraft model, derivation of the first order necessary conditions and derivation of second order necessary condition are given in Appendices A, B, C, and D respectively.

\section{Modeling}

In this section the model used throughout the paper is presented. The model consists of three parts: the aircraft kinematic model, the energy collection model and the energy loss model. Each subsystem is presented below.

\section{II.A. Aircraft Kinematic Model}

The bank-to-turn aircraft is assumed to fly in still air and remain at constant altitude, with zero pitch angle, according to the equations:

$$
\begin{aligned}
\dot{x} & =V \cos \psi, \\
\dot{y} & =V \sin \psi, \\
\dot{\psi} & =\frac{g \tan \phi}{V},
\end{aligned}
$$

where $x$ and $y$ are the Cartesian coordinates of the aircraft, $\psi$ is the heading angle, $V$ is the speed and $\phi$ is the bank angle.

\section{II.B. Energy Collection Model}

The aircraft is equipped with solar cells, mounted on the top side of the wings, and gains solar energy from the sun shining on the cells. Let $a$ and $e$ represent the azimuth and elevation angles of the sun, respectively. Assuming that the wing configuration has zero dihedral angle, the incidence angle of the sun rays upon the solar cells, $i$, satisfies:

$$
\cos (i)=\cos (\phi) \sin (e)-\cos (e) \sin (a-\psi) \sin (\phi),
$$

which is derived in Appendix A. In this paper we assume that the sun is fixed in the sky. Equation (4) holds, however, even if the sun moves. The power collected by the aircraft is:

$$
P_{\text {in }}=\eta_{s o l} P_{s d} S \cos (i),
$$

where $\eta_{s o l}$ is the efficiency of the solar cell, $P_{s d}$ is the solar spectral density, and $S$ is the total surface area of the wing. If less than a full wing is to be covered by solar cells, $\eta_{s o l}$ can be adjusted to account for this decrease in solar cell area.

During a time interval $\left[t_{o}, t_{f}\right]$, the energy collected by the aircraft is:

$$
E_{i n}=\int_{t_{o}}^{t_{f}} P_{i n}(t) d t
$$

\section{II.C. Energy Loss Model}

Energy lost by the craft is derived from standard lift, drag and propulsion models (See Ref. 53). The constant altitude assumption requires $L \cos (\phi)=W$ where $L$ is lift and $W$ is the weight of the aircraft. The equations governing the power lost driving the propeller, $P_{\text {out }}$, are: 


$$
\begin{aligned}
C_{L} & =\frac{2 W}{\rho V^{2} S \cos \phi}, \\
C_{D} & =C_{D_{O}}+K C_{L}^{2}, \\
D & =1 / 2 \rho V^{2} S C_{D}, \\
T & =D, \\
P_{\text {out }} & =\frac{T V}{\eta_{\text {prop }}},
\end{aligned}
$$

where $C_{L}$ is the coefficient of lift, $\rho$ is the air density, $C_{D}$ is the coefficient of drag, $C_{D_{O}}$ is the parasitic drag, the aerodynamic coefficient $K$ represents the amount whereby the induced drag exceeds that of an elliptical lift distribution, $\eta_{\text {prop }}$ is the efficiency of the propeller and $T$ is the thrust of the aircraft. The numerical values of the aircraft parameters used in this paper are presented in Appendix B.

During a time interval $\left[t_{o}, t_{f}\right]$, the energy lost by the aircraft is:

$$
E_{\text {out }}=\int_{t_{o}}^{t_{f}} P_{\text {out }}(t) d t
$$

\section{II.D. Model Summary}

In summary, the integrated model is as follows. The bank angle determines the heading and the position of the aircraft through (1)-(3). This bank angle, combined with the sun's position determines the incidence angle through (4). The incidence angle of the sun together with the bank angle and speed determine the energy collected and lost by the aircraft during flight through (5)-(6) and (7)-(12), respectively.

\section{Problem Formulation}

\section{III.A. Mission Description}

The missions considered in this paper are to fly from given initial location and heading $\left(x_{o}, y_{o}, \phi_{o}\right)$ to given final location and heading $\left(x_{f}, y_{f}, \phi_{f}\right)$ within a time interval $\left[t_{o}, t_{f}\right]$. In a typical mission, $t_{o}$ is given, and $t_{f}$ must satisfy $t_{f} \leq t_{o}+T_{M}$, where $T_{M}$ is given and represents the maximum duration of the mission. This duration is assumed short compared to the duration of daylight, so that the solar spectral density and sun position are assumed constant during the mission.

\section{III.B. Dynamic Optimization Problem}

The dynamic optimization problem presented in this paper is motivated by the requirement to maximize, with respect to the time-histories of the bank angle and speed, the final energy of the solar-powered aircraft, i.e.,

$$
\begin{aligned}
\max _{\phi(\cdot), V(\cdot)}\left(E_{\text {in }}-E_{\text {out }}\right) \\
\quad \text { subject to (1)-(12) and boundary conditions. }
\end{aligned}
$$

In practice, a larger value of objective function (13) increases the endurance of the aircraft.

\section{Optimal Path Planning}

In this section, we derive the necessary conditions for optimality that characterize the optimal trajectories.

The necessary conditions for optimality for the maximization problem (1)-(12) are derived in Ref. 46. Here, these necessary conditions are applied to the current problem. With states $[x, y, \psi]^{T}$ and control inputs $(\phi, V)^{T}$ the Hamiltonian is:

$$
H\left(x, y, \psi, \lambda_{x}, \lambda_{y}, \lambda_{\psi}, \phi, V\right)=P_{\text {in }}-P_{\text {out }}+\lambda_{x} V \cos \psi+\lambda_{y} V \sin \psi+\lambda_{\psi} \frac{g \tan \phi}{V},
$$


where $\lambda_{x}, \lambda_{y}$, and $\lambda_{\psi}$ are the costates. In this problem, the only control constraints are that $|\phi|<\frac{\pi}{2}$ and $V>0$. While velocity is constrained by performance of the engine, altitude, etc, it is assumed that the aircraft can at least fly at the minimum-power velocity (Ref. 53):

$$
V_{\text {Power }_{\text {min }}}=\sqrt[4]{\frac{4 K W^{2}}{3 C_{D_{o}} \rho^{2} S^{2} \cos ^{2}(\phi)}} .
$$

We also assume that

$$
\frac{\sqrt{\left(x_{f}-x_{o}\right)^{2}+\left(y_{f}-y_{o}\right)^{2}}}{T_{M}} \leq V_{\text {Power }_{\text {min }}},
$$

in other words, if the UAV were to fly on a straight path between the initial and final locations, and at minimum-power velocity, it would complete the mission within the allowed duration.

In our experience, it is not necessary to impose a tight constraint on the magnitude of the bank angle. Indeed, banking requires lifting (See Eq. (7)), lifting induces drag (See Eq. (8)), drag requires thrust (See Eq. (10)), which implies power loss (See Eq. (11)). Since the path planning aims at achieving optimal final energy, the magnitude of the bank angle is naturally limited by these phenomena.

The state equations, derived from (14), are:

$$
\begin{aligned}
& \dot{x}=\frac{\partial H}{\partial \lambda_{x}}=V \cos (\psi), \\
& \dot{y}=\frac{\partial H}{\partial \lambda_{y}}=V \sin (\psi), \\
& \dot{\psi}=\frac{\partial H}{\partial \lambda_{\psi}}=\frac{g \tan (\phi)}{V} .
\end{aligned}
$$

The costate equations are:

$$
\begin{gathered}
\dot{\lambda_{x}}=\frac{-\partial H}{\partial x}=0, \\
\dot{\lambda_{y}}=\frac{-\partial H}{\partial y}=0, \\
\dot{\lambda_{\psi}}=\frac{-\partial H}{\partial \psi}=-\lambda_{y} V \cos \psi+\lambda_{x} V \sin \psi-\eta_{s o l} P_{s d} S \cos e \cos (a-\psi) \sin \phi .
\end{gathered}
$$

The first-order optimality conditions are:

$$
\begin{aligned}
& \frac{\partial H}{\partial \phi}=-\eta_{\text {sol }} P_{s d} S_{c}(\cos (e) \cos (\phi) \sin (a-\psi)+\sin (e) \sin (\phi))-\frac{4 K W^{2} \sin (\phi)}{\eta_{\text {prop }} \rho S V \cos ^{3}(\phi)}+\frac{g \lambda_{\psi}}{V \cos ^{2}(\phi)}=0, \\
& \frac{\partial H}{\partial V}=\lambda_{x} \cos (\psi)+\frac{8 K W^{2} \sec (\phi)^{2}}{\eta_{\text {prop }} \rho S V^{2}}-\frac{3 \rho S V^{2}\left(C_{D_{o}}+\frac{4 K W^{2} \sec (\phi)^{2}}{\rho^{2} S^{2} V^{4}}\right.}{2 \eta_{\text {prop }}}+\lambda_{y} \sin (\psi)-\frac{g \lambda_{\psi} \tan (\phi)}{V^{2}}=0 .
\end{aligned}
$$

The second order Legrendre-Clebsch condition is that the Hessian of the Hamiltonian be negative semidefinite, i.e.:

$$
\frac{\partial^{2} H}{\partial(\phi, V)^{2}} \leq 0
$$

where, if

$$
\frac{\partial^{2} H}{\partial(\phi, V)^{2}}=\left[\begin{array}{cc}
H_{\phi \phi} & H_{\phi V} \\
H_{\phi V} & H_{V V}
\end{array}\right]
$$




$$
\begin{aligned}
H_{\phi \phi} & =\frac{\eta_{\text {prop }} \eta_{s o l} \rho P_{s d} S^{2} V(-(\cos (\phi) \sin (e))+\cos (e) \sin (a-\psi) \sin (\phi))}{\eta_{\text {prop }} \rho S V}+\frac{-4 K W^{2} \sec (\phi)^{4}+2 \sec (\phi)^{3}\left(g \lambda_{\psi} \eta_{p r o p} \rho S \cos (\phi)-4 K W^{2} \sin (\phi)\right) \tan (\phi)}{\eta_{\text {prop }} \rho S V}, \\
H_{\phi V} & =-\frac{g \lambda_{\psi} \sec (\phi)^{2}}{V^{2}}+\frac{4 K W^{2} \sec (\phi)^{2} \tan (\phi)}{\eta_{p r o p} \rho S V^{2}}, \\
H_{V V} & =\frac{8 K W^{2} \sec (\phi)^{2}}{\eta_{\text {prop }} \rho S V^{3}}-\frac{3 \rho S V\left(C_{D_{o}}+\frac{4 K W^{2} \sec (\phi)^{2}}{\rho^{2} S^{2} V^{4}}\right)}{\eta_{\text {prop }}}+\frac{2 g \lambda_{\psi} \tan (\phi)}{V^{3}} .
\end{aligned}
$$

The boundary conditions for this problem are:

$$
\begin{aligned}
x\left(t_{o}\right) & =x_{o}, \\
y\left(t_{o}\right) & =y_{o}, \\
\psi\left(t_{o}\right) & =\phi_{o}, \\
x\left(t_{f}\right) & =x_{f}, \\
y\left(t_{f}\right) & =y_{f}, \\
\psi\left(t_{f}\right) & =\phi_{f} .
\end{aligned}
$$

If the final time is free, then we must satisfy

$$
H\left(t_{f}\right)=0
$$

However, if the final time is fixed, Eq. (34) does not necessarily hold.

Equations (17)-(34) provide necessary conditions for optimality in the form of a two-point boundary value problem. By choosing an initial state $\left(x_{o}, y_{o}, \phi_{o}\right)^{T}$ and an initial costate $\left(\lambda_{x o}, \lambda_{y o}, \lambda_{\psi o}\right)^{T}$, we can numerically integrate the differential equations (17)-(22) subject to (23) and (24). This results in a flight path that satisfies the first order necessary conditions, and along which the second order condition (25) can easily be checked. We will call this strategy the numerical integration method.

We will refer to the flight paths that satisfy the first and second order necessary conditions (17)-(34) as extremal paths.

\section{The Discretization Procedure}

\section{V.A. Discretization}

To obtain numerical approximations of optimal paths, we discretize the problem as follows. For a chosen integer $n \geq 1$, we subdivide the interval $\left[t_{o}, t_{f}\right]$ into $n$ subintervals $\left[t_{o}, t_{1}\right],\left[t_{1}, t_{2}\right], \ldots,\left[t_{n-1}, t_{f}\right]$ of equal duration. In each subinterval we assume that the control input is constant, i.e., $(\phi(t), V(t))=\left(\phi_{j}, V_{j}\right), t \in\left[t_{j}, t_{j+1}\right]$, where the parameters $\left(\phi_{j}, V_{j}\right), 0 \leq j \leq n-1$, are unknown.

We treat the parameters $\left(\phi_{j}, V_{j}\right), 0 \leq j \leq n-1$, and $t_{f}$ as inputs to a nonlinear optimization problem. As an initial choice, in all subintervals we choose $\phi_{j}=0, V_{j}=V_{\text {Powermin }_{\text {min }}}$ and $t_{f}=t_{o}+T_{M}$. Constraints upon this problem are imposed from the boundary conditions (28)-(33). From (13), the objective function is the total energy at the end of flight. We then numerically solve for optimal flight paths using the MATLAB ${ }^{\circledR}$ Optimization Toolbox function fmincon and the ordinary differential equation solver ode45. We will call this strategy the discretization method.

\section{V.B. Characteristics of Discretization Results}

The same set of simulation conditions was used for each result presented in this paper. These conditions are shown in table 1.

Table 1. Simulation Conditions

\begin{tabular}{lccc}
\hline Initial Position & $\left(x_{o}, y_{o}\right)$ & $(0,0)$ & $\mathrm{m}$ \\
Initial Energy & $E_{o}$ & 0 & $\mathrm{~J}$ \\
Initial Heading & $\phi_{o}$ & 127 & $\mathrm{deg}$ \\
Final Position & $\left(x_{f}, y_{f}\right)$ & $(700,1300)$ & $\mathrm{m}$ \\
Maximum Duration of Flight & $T_{M}$ & 300 & $\mathrm{sec}$ \\
Velocity of Minimum Power & $V_{\text {Power }_{\text {min }}}$ & 15 & $\mathrm{~m} / \mathrm{s}$ \\
\hline
\end{tabular}




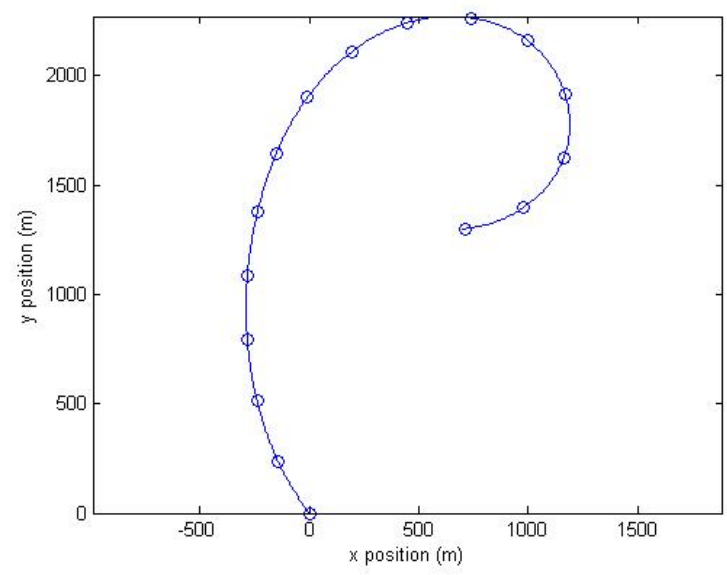

(a) Flight Path

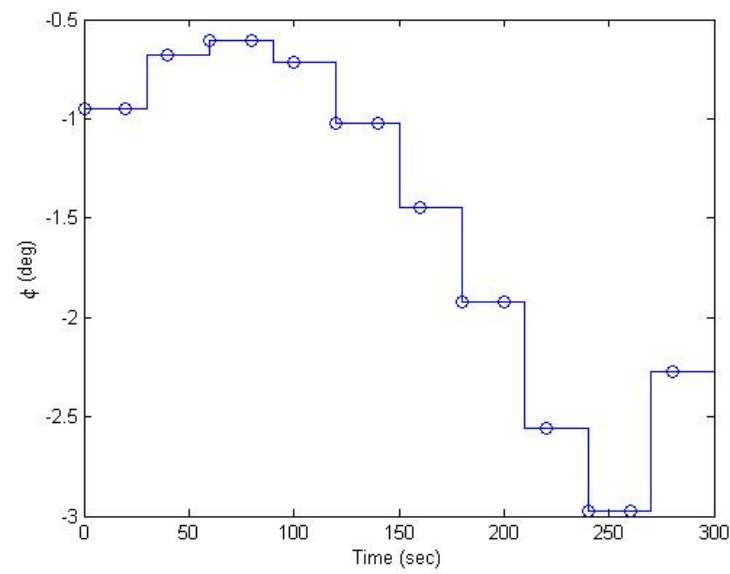

(b) Bank Angle

Figure 1. Example of an energy optimal flight path based on table 2. Dots are placed every 20 sec.

Figures 1 and 2 are representative samples of optimal flight paths, created using the discretization method. Each flight path was then evaluated based upon the time of flight and total energy at the end of flight. A summary of the conditions and results for figure 1 is presented in table 2 .

Of particular note, in figure 1 , is the positive total energy at the end of flight, indicating a net gain of energy. The flight duration is equal to $T_{M}$, the maximum allowed. The final time, $t_{f}$, is free in this problem subject to $t_{f} \leq t_{o}+T_{M}$. The aircraft also only made slow, sweeping turns with $\left|\phi_{j}\right|<<1$ for all $j$. Throughout the flight the speed remained a constant $15 \mathrm{~m} / \mathrm{s}$.

A summary of the conditions and results for figure 2 is presented in table 3 . The only difference between the environment settings is that the sun has set in this simulation. During this flight, the bank angle is close to $0 \mathrm{deg}$, indicating almost no turning. The only turn that did occur was at the beginning of flight to obtain a direct heading towards the destination. The control parameters during this turn indicate a high speed and high bank angle for a short duration. The total flight duration is only $80 \mathrm{sec}$, much less than $T_{M}$. In this case, the total energy during the flight was negative, indicating more energy was lost than collected. The speed throughout the flight remained at a constant $15 \mathrm{~m} / \mathrm{s}$.

As shown in figures 1 and 2, the flight path characteristics vary widely as the elevation is changed. Figure 
Table 2. Figure 1 Simulation Conditions and Results

\begin{tabular}{lccc}
\hline Sun Position & $(a, e)$ & $(0,45)$ & $\mathrm{deg}$ \\
Solar Spectral Density & $P_{s d}$ & 380 & $W / \mathrm{m}^{2}$ \\
Final Heading & $\phi_{f}$ & 270 & $\mathrm{deg}$ \\
Flight Duration & $t_{f}-t_{o}$ & 300 & $\mathrm{sec}$ \\
Total Final Energy & $E_{T}$ & 6764 & $\mathrm{~J}$ \\
Energy In & $E_{\text {in }}$ & 12646 & $\mathrm{~J}$ \\
\hline
\end{tabular}

Table 3. Figure 2 Simulation Conditions and Results

\begin{tabular}{lccc}
\hline Sun Position & $(a, e)$ & $(0,0)$ & $\mathrm{deg}$ \\
Solar Spectral Density & $P_{s d}$ & 380 & $W / \mathrm{m}^{2}$ \\
Final Heading & $\phi_{f}$ & 61 & $\mathrm{deg}$ \\
Flight Duration & $t_{f}-t_{o}$ & 80 & $\mathrm{sec}$ \\
Total Final Energy & $E_{T}$ & -1776.1 & $\mathrm{~J}$ \\
Energy In & $E_{i n}$ & 554.7 & $\mathrm{~J}$ \\
\hline
\end{tabular}

3 is obtained by varying the elevation of the sun and recording the total energy of the resulting optimal flight path while the remaining aircraft, environmental and mission parameters are fixed. The elevation is varied from 0 to 90 degrees. While the total energy of the aircraft at the end of flight remains positive, a sinusoidal relationship between elevation and energy emerges. This sinusoid may be approximated by fitting a curve as:

$$
E_{T}=11970 \sin \left(\frac{90}{72} e\right) .
$$

This relationship persists until the total energy becomes negative. At this energy transition point, the trend departs from a sinusoidal function, which suggests a change in regime. The dashed line in the figure represents the total energy acquired during a straight flight directly towards the final position.

\section{V.C. Insights from Discretization Results}

From the discretization results, we formulate the following propositions describing the observed characteristics in figures 1-3. Let $E_{\text {Total }}$ denote the cost functional in (13) when using a straight unbanked flight path between the initial location $\left(x_{o}, y_{o}\right)$ and the final location $\left(x_{f}, y_{f}\right)$.

Proposition 1 If $P_{\text {sd }}$ is small enough, then $\phi(t)=0, V=V_{\text {Power }_{\text {min }}}$ generate a path that satisfies the necessary conditions for optimal flight, and $t_{f} \leq t_{o}+T_{M}$.

Note that, in practice, $P_{s d}$ small implies $E_{\text {Total }}<0$. However, Proposition 1 does not mean that $E_{\text {Total }}<0$ implies $\phi(t)=0, V=V_{\text {Power }_{\text {min }}}$ generate the only path that satisfies the necessary conditions of optimality. We will show additional paths satisfying the necessary conditions where $E_{\text {Total }}<0$ and $\phi(t) \neq 0$ later in this paper.

Proposition 2 If $E_{\text {Total }}>0$, and $T_{M}$ and $E_{\text {Total }}$ are large enough, then the optimal path must satisfy $t_{f}=t_{o}+T_{M}$ and $V=V_{\text {Power }_{\text {min }}}$.

Proposition 2 implies that when $E_{\text {Total }}>0$ and large enough, and $T_{M}$ is large enough, the optimal path takes as much time as allowed, flying at the most advantageous speed. Propositions 1 and 2 will be proved in Section VII, based on experimental results presented in Section VI.

\section{The Power Ratio}

The simulation results and insights presented depend upon the sign of $E_{\text {Total }}$. If $E_{\text {Total }}<0$ we have on average that $P_{\text {in }}<P_{\text {out }}$. Conversely, if $E_{\text {Total }}>0$ we have on average $P_{\text {in }}>P_{\text {out }}$. These results may be 


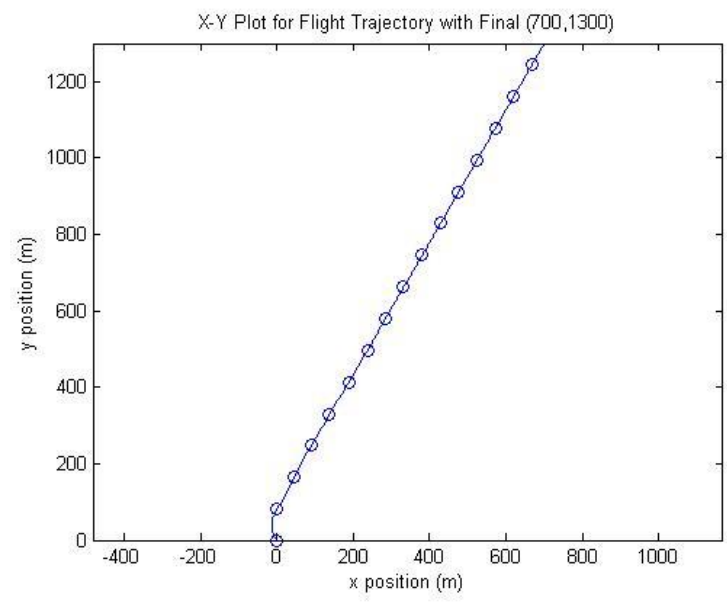

(a) Flight Path

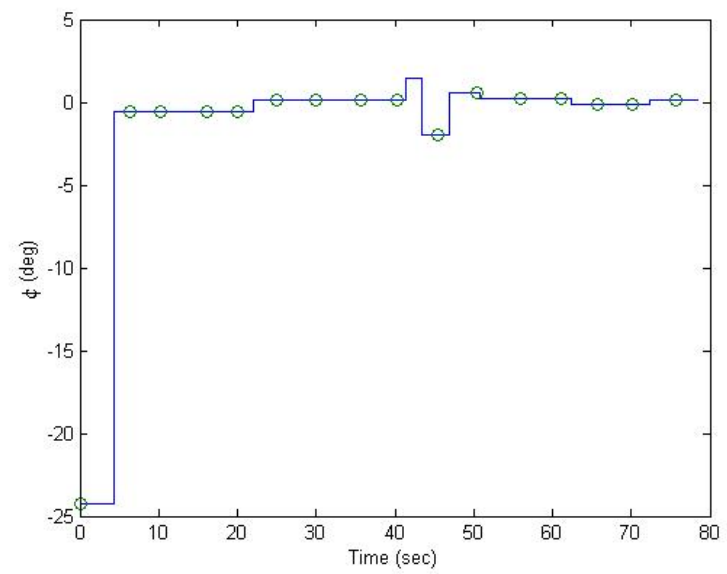

(b) Bank Angle

Figure 2. Example of an energy optimal flight path based on table 3. Dots are placed every 5 sec.

expressed as $\frac{P_{\text {in }}}{P_{\text {out }}}<1$ or $\frac{P_{\text {in }}}{P_{\text {out }}}>1$, respectively. When considering a straight unbanked flight path between the initial location and the final location, as in Proposition 1, this fraction may be expressed as:

$$
P_{R}=\frac{2 \eta_{\text {prop }} \eta_{\text {sol }} \rho P_{s d} S^{2} V_{\text {Power }_{\text {min }}} \sin (e)}{C_{D_{O}} \rho^{2} S^{2} V_{\text {Power }_{\text {min }}}^{4}+4 K W^{2}}
$$

We refer to this non-dimensional number as $P_{R}$, the Power Ratio.

During flight, $P_{\text {in }}$ and $P_{\text {out }}$ become functions of the bank angle, $\phi$, heading angle, $\psi$, and the azimuth of the sun, $a$. At the end of flight, we can evaluate the total energy collected and lost, and compute the energy ratio, $E_{R}$, as: 


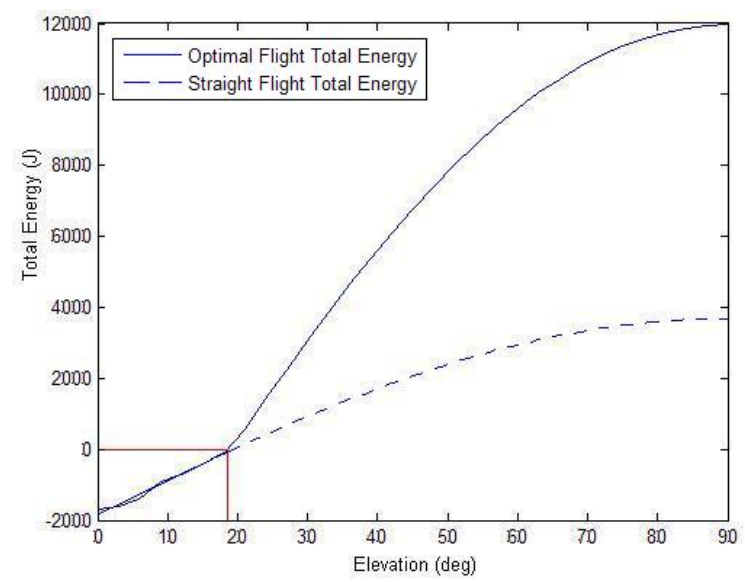

Figure 3. Total energy at end of flight as a function of solar elevation

$$
E_{R}=\frac{\int_{t_{o}}^{t_{f}} P_{\text {in }}(t) d t}{\int_{t_{o}}^{t_{f}} P_{\text {out }}(t) d t} .
$$

$E_{R}$ defines the two flight regimes observed, which we call the Drag Regime and the Solar Regime, corresponding to $E_{R}<1$ and $E_{R}>1$, respectively.

Because $P_{R}$ does not depend on specific values of $(\phi, \psi, a)$, we can use it to predict the performance of the aircraft before flight. In order to validate this usage, table 4 compares $P_{R}$ with $E_{R}$, the Energy Ratio computed after a variety of flights. It turns out that $P_{R}$ exactly matches $E_{R}$ under the conditions specified by Proposition 1. Therefore table 4 only examines those flights when $E_{R}>1$.

Table 4. Typical Comparison of $P_{R}$ and $E_{R}$ on Energy-Optimal Paths

\begin{tabular}{ccc}
\hline$P_{R}$ & $E_{R}$ & Error \\
2.3492 & 2.3511 & $-0.08 \%$ \\
2.2016 & 2.2125 & $-0.49 \%$ \\
2.0771 & 2.0745 & $0.13 \%$ \\
1.9112 & 1.9307 & $-1.01 \%$ \\
1.7704 & 1.7822 & $-0.66 \%$ \\
1.6304 & 1.6226 & $0.48 \%$ \\
1.4810 & 1.4548 & $1.80 \%$ \\
1.3149 & 1.2983 & $1.28 \%$ \\
1.1526 & 1.1551 & $-0.22 \%$ \\
1.0068 & 1.0041 & $0.27 \%$ \\
\hline
\end{tabular}

In summary, if $P_{R}$ is evaluated for a specified mission before flight, a non-dimensional value emerges. The Energy Ratio of the optimal flight path closely approximates this value. Thus, we have the following:

Experimental Fact $1 P_{R}$ can be used instead of $E_{R}$ to determine the regime of the optimal flight.

\section{Properties of Extremal Paths}

So far, Propositions 1 and 2 were inferred from observed characteristics of numerical approximations of optimal flight paths. We will now prove them through application of the necessary conditions (16)-(24), using Experimental Fact 1. 


\section{VII.A. Proof of Proposition 1}

We first assume that $\phi(t)=0$, simplify the necessary conditions and solve for the states and costates subject to the constraints (28)-(34). This results in:

$$
\begin{gathered}
\phi=0, \\
V=V_{\text {Power }_{\text {min }},}, \\
\psi=\arctan \left(\frac{y_{f}-y_{o}}{x_{f}-x_{o}}\right), \\
t_{f}=\frac{x_{f}-x_{o}}{\cos \psi V}=\frac{y_{f}-y_{o}}{\sin \psi V}, \\
\lambda_{x}=0 \\
\lambda_{y}=0 \\
\lambda_{\psi}=\frac{V \eta_{s o l} P_{s d} S \cos (e) \sin (a-\psi)}{g},
\end{gathered}
$$

where each of the costates is constant. Here, both $V$ and $\psi$ remain constant at their initial values. A derivation of (39)-(44) is provided in Appendix C. The flight duration depends on the distance to the final destination and the velocity, and is feasible because of (16).

It is shown in Appendix D that the flight conditions (38)-(44) satisfy the second order necessary condition for optimality. Since $\phi=0$ yields a path that satisfies the necessary conditions for optimal flight, we have proven Proposition 1.

\section{VII.B. Proof of Proposition 2}

Since $E_{\text {Total }}>0$, the optimal path must satisfy $E_{\text {in }}-E_{\text {out }}>0$. From Experimental Fact 1, this yields $P_{\text {in }}-P_{\text {out }}>0$. For $E_{\text {Total }}$ large enough, $P_{\text {in }}-P_{\text {out }}$ will be large enough to be the dominant term in the right hand side of (14). Therefore, $H>0$ when $E_{\text {Total }}>0$ and large enough.

From Ref. 46, we may express the transversality conditions as:

$$
\left[\lambda^{T} \delta x-H \delta t\right]_{t_{o}}^{t_{f}}=0
$$

or, when focusing on the variation of the final time,

$$
H\left(t_{f}\right) \delta t_{f}=0 .
$$

Since $H>0, \delta t_{f}=0$ which implies that the final time is fixed. Therefore $t_{f}=t_{o}+T_{M}$, which proves the first claim.

We must now examine the velocity of the aircraft when $E_{\text {Total }}>0$. Consider an extremal path that

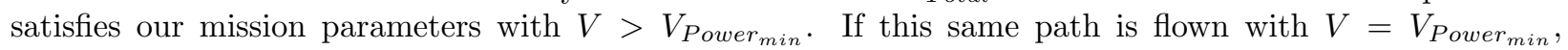
$E_{\text {Total }}$ will be higher than with $V>V_{\text {Power }_{\text {min }}}$. Since velocity is constrained to be greater than or equal to $V_{\text {Power }_{\text {min }}}$, a maximum $E_{\text {Total }}$ occurs when $V=V_{\text {Power }_{\text {min }}}$. Thus $V=V_{\text {Power }_{\text {min }}}$ is optimal and we have proven the second claim and Proposition 2.

\section{VII.C. Special Properties when $P_{R}<1$}

Numerical integration of the necessary conditions (17)-(24) has shown that there are curved paths that are extremal with $P_{R}<1$. Since we have already shown that Proposition 1 holds when $P_{R}<1$, we may compare a flight path formed from integrating (17)-(24) with a similar flight path solution satisfying Proposition 1. Figure 4 illustrates a comparison of such flight paths.

While the flight path satisfying (17)-(24) has a $P_{R}$ value of 0.7402 , the flight path satisfying Proposition 1 has a $P_{R}$ value of 0.7418 . This slightly larger $P_{R}$ suggests an increased endurance for the aircraft. From this result we conjecture that, while both flight paths are extremal, the flight path satisfying Proposition 1 is a global maximizer while the curved extremal is only a local maximizer. 


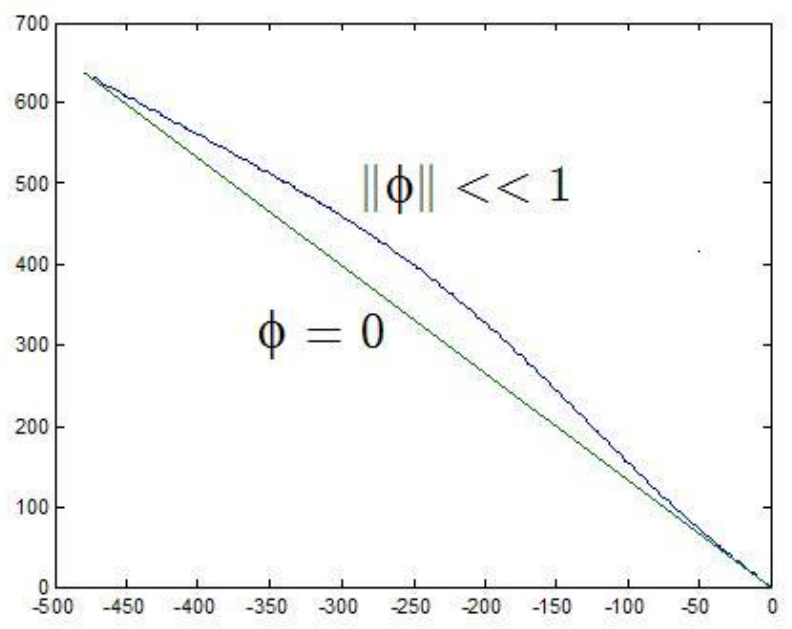

Figure 4. Extremal Paths when $P_{R}>1$

\section{VII.D. Extremal Path Summary}

In summary, extremal flight paths can be obtained as follows. When $P_{R}<1$, extremal flight paths are best described by Proposition 1. When $P_{R}>1$, extremal flight paths satisfy Proposition 2 . The velocity in both regimes is not an independent control but is instead dependent upon the bank angle.

\section{Design Implications of the Power Ratio}

We can manipulate $P_{R}$ to clarify the distinct roles of environmental parameters and aircraft parameters. By substituting in for $V$ the full expression (15) for $V_{P_{\text {Power }} \text { in }}$, we simplify $P_{R}$ as:

$$
P_{R}=0.402 P_{s d} \eta_{s o l} \sin (e) \sqrt{\rho} \sqrt[4]{\frac{\eta_{p r o p}^{4} b^{6} \epsilon^{3} \pi^{3} S^{3}}{C_{D_{o}} W^{6}}},
$$

where $\epsilon$ is the Oswald efficiency factor and $b$ is the wingspan. The form of $P_{R}$ in (47) separates those terms depending on the environment, $\left[P_{s d}, \eta_{s o l}, e, \rho, g\right]$, and those depending on the airframe, $\left[\eta_{\text {prop }}, b, \epsilon, S, C_{D_{o}}, m\right]$. From (47), a very long wing with a large surface area is advantageous for solar-powered flight. As expected, most successful solar-powered UAVs do indeed have these characteristics - see, e.g., Helios (Ref. 31), Solong (Ref. 20), Pathfinder (Ref. 9), or Sunriser (Ref. 15). A more detailed utilization of the power ratio for design is presented in Ref. 54 .

\section{Summary, Conclusions and Future Work}

We have presented a new integrated model for optimal energy path planning for solar powered UAVs. The model accounts for the coupling of the kinematics with the energy collected and lost. This coupling happens through the bank angle of the aircraft. Based on this model, we have presented necessary conditions for the maximization of total energy and proven satisfaction of these necessary conditions in two different regimes of flight. We have shown that a prediction of the optimal regime of flight can be made accurately before flight and that this optimal regime drastically affects the path (direct or loitering) taken by the UAV. The determination of this optimal regime of flight is made through the Power Ratio. Further results suggest that the Power Ratio can be used for design of aircraft.

This paper has experimentally shown that the Power Ratio is approximately equal to the Energy Ratio. The Power Ratio is also a determining factor for the choice between two optimal regimes of flight. By manipulating $P_{R}$, we have shown that the similarity in looks of solar-powered UAVs is not accidental, indeed, it is systemic of efficient design. Most importantly, this paper has shown that the energy collected by a solar powered UAV can be increased both through efficient design and optimal path planning. 
In future work, we will find sufficient conditions for optimality that characterize optimal energy trajectories. We will consider optimal energy path planning in non-level flight conditions and also validate Propositions 1 and 2 through a series of flight tests of a solar powered UAV.

\section{Appendix A: Derivation of the Solar Incidence Angle}

Define $[g]=\left[\hat{x}_{g}, \hat{y}_{g}, \hat{z}_{g}\right]^{T}$ as a vectrix (See Ref. 49) fixed to the ground with $\hat{z}_{g}$ vertical ascending. If $a$ and $e$ are the azimuth and elevation of the sun, then $\hat{s}$, the unit vector to the sun, is given as

$$
\hat{s}=[g]^{T}\left[\begin{array}{c}
\cos (e) \cos (a) \\
\cos (e) \sin (a) \\
\sin (e)
\end{array}\right] .
$$

Define $[a]=\left[\hat{x}_{a}, \hat{y}_{a}, \hat{z}_{a}\right]^{T}$ as a vectrix fixed to the aircraft. In terms of $[g]$,

$$
[a]=R_{1}(\phi) R_{3}(\psi)[g]
$$

where,

$$
\begin{aligned}
R_{1}(\phi) & =\left[\begin{array}{ccc}
1 & 0 & 0 \\
0 & \cos (\phi) & \sin (\phi) \\
0 & -\sin (\phi) & \cos (\phi)
\end{array}\right], \\
R_{3}(\psi) & =\left[\begin{array}{ccc}
\cos (\psi) & \sin (\psi) & 0 \\
-\sin (\psi) & \cos (\psi) & 0 \\
0 & 0 & 1
\end{array}\right],
\end{aligned}
$$

represent rotation matrices about the first and third axis, respectively. By inverting this relationship, we obtain

$$
[g]=R_{3}(\psi)^{T} R_{1}(\phi)^{T}[a] .
$$

Hence, $\hat{s}$ can be expressed, in the aircraft-fixed vectrix, as

$$
\hat{s}=\left[\begin{array}{c}
\cos (e) \cos (a) \\
\cos (e) \sin (a) \\
\sin (e)
\end{array}\right] R_{3}(\psi)^{T} R_{1}(\phi)^{T}[a] .
$$

Define the incidence angle $i$ as the angle between the line-of-sight to the sun and the $\hat{z}$-axis of the aircraft-fixed vectrix. Then $i=\arccos \left(\hat{s} \cdot \hat{z}_{a}\right)$. Hence, (53) yields:

$$
\cos (i)=\cos (e) \cos (a) \sin (\psi) \sin (\phi)-\cos (e) \sin (a) \cos (\psi) \sin (\phi)+\sin (e) \cos (\phi),
$$

or, after applying trigonometric identities,

$$
\cos (i)=\sin (e) \cos (\phi)-\cos (e) \sin (\phi) \sin (a-\psi) .
$$

\section{Appendix B: Aircraft Model Parameters}

The aircraft model used in all simulations and numerical results in this paper was developed by the University of Michigan SolarBubbles Team (See Refs. 50-52). Aerodynamic coefficients were evaluated through the use of AVL and Fluent and verified experimentally through full size wind tunnel testing (Ref $52)$.

The flying wing aircraft, pictured in figure 5 , has the characteristics listed in table 5 . 
Table 5. Aircraft Model Parameters

\begin{tabular}{lccc}
\hline Wing Area & $S$ & 0.1566 & $\mathrm{~m}^{2}$ \\
Mass & $m$ & 1.2 & $\mathrm{~kg}$ \\
Wingspan & $b$ & 0.711 & $\mathrm{~m}$ \\
Oswald Efficiency Factor & $\epsilon$ & 0.992 & \\
Parasitic Drag & $C_{D_{o}}$ & 0.011 & \\
Propeller Efficiency & $\eta_{\text {prop }}$ & 0.7 & \\
Air Density & $\rho$ & 1.29 & $\mathrm{~kg} / \mathrm{m}^{3}$ \\
\hline
\end{tabular}

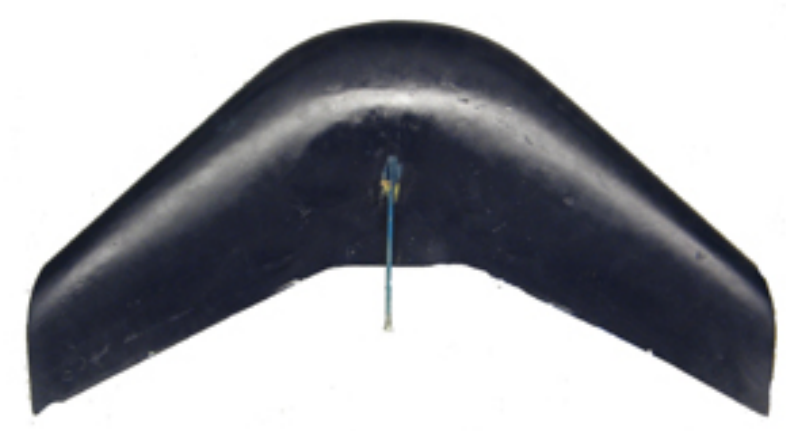

Figure 5. Wind Tunnel Aircraft Model

\section{Appendix C: Derivation of Drag Regime First Order Necessary Conditions}

The first order necessary conditions, simplified as in Proposition 1, are:

$$
\begin{aligned}
\frac{\partial H}{\partial \phi}=-\eta_{s o l} P_{s d} S_{c} \cos (e) \sin (a-\psi)+\frac{g \lambda_{\psi}}{V}=0 & \\
\frac{\partial H}{\partial V}=\lambda_{x} \cos (\psi)+\frac{8 K W^{2}}{\eta_{p r o p} \rho S V^{2}} & -\frac{3 \rho S V^{2}\left(C_{D_{o}}+\frac{4 K W^{2}}{\rho^{2} S^{2} V^{4}}\right)}{2 \eta_{p r o p}}+\lambda_{y} \sin (\psi)=0 \\
\dot{x} & =\frac{\partial H}{\partial \lambda_{x}}=V \cos (\psi), \\
\dot{y} & =\frac{\partial H}{\partial \lambda_{y}}=V \sin (\psi), \\
\dot{\psi} & =\frac{\partial H}{\partial \lambda_{\psi}}=0 \\
\dot{\lambda_{x}} & =\frac{-\partial H}{\partial x}=0 \\
\dot{\lambda_{y}} & =\frac{-\partial H}{\partial y}=0 \\
\dot{\lambda_{\psi}}=\frac{-\partial H}{\partial \psi} & =-\lambda_{y} V \cos \psi+\lambda_{x} V \sin \psi
\end{aligned}
$$

Since $\dot{\lambda_{x}}, \dot{\lambda_{y}}$, and $\dot{\psi}$ are all zero, $\lambda_{x}, \lambda_{y}$ and $\psi$ are all constants. Equation (56) becomes a function of constant parameters and $V$. From this we can see that $V$ must be constant as well. Similarly, (56) is now a 
function of constant parameters and $\lambda_{\psi}$. Accordingly, $\lambda_{\psi}$ must also be constant, and:

$$
\dot{\lambda_{\psi}}=-\lambda_{y} V \cos \psi+\lambda_{x} V \sin \psi=0 .
$$

Now (56) yields:

$$
\lambda_{\psi}=\frac{\eta_{s o l} P_{s d} V S \cos (e) \sin (a-\psi)}{g} .
$$

We can now solve for $\lambda_{x}$ and $\lambda_{y}$ using (58) and (65) as:

$$
\begin{gathered}
\lambda_{x}=\frac{\left(-4 K W^{2}+3 C_{D_{O}} \rho^{2} S^{2} V^{4}\right) \cos \psi}{2 \eta_{\text {prop }} \rho S V^{2}}, \\
\lambda_{y}=-\frac{\left(-4 K W^{2}+3 C_{D_{O}} \rho^{2} S^{2} V^{4}\right) \sin \psi}{2 \eta_{\text {prop }} \rho S V^{2}} .
\end{gathered}
$$

Equations (57), (66) and (67) are satisfied simultaneously by $\lambda_{x}=0, \lambda_{y}=0$ and $V=\sqrt[4]{\frac{4 K W^{2}}{3 C_{D_{o} \rho^{2} S^{2}}}}$, which is the velocity at minimum power

\section{Appendix D: Satisfaction of the Drag Regime Second Order Necessary Condition}

The second order necessary condition is:

$$
\frac{\partial^{2} H}{\partial \phi^{2}} \leq 0
$$

where, if

$$
\frac{\partial^{2} H}{\partial \phi^{2}}=\left[\begin{array}{cc}
\alpha & \beta \\
\beta & \gamma
\end{array}\right]
$$

$\alpha=\frac{\eta_{\text {prop }} \eta_{\text {sol }} \rho P_{s d} S^{2} V(-(\cos (\phi) \sin (e))+\cos (e) \sin (a-\psi) \sin (\phi))}{\eta_{\text {prop }} \rho S V}+\frac{-4 K W^{2} \sec (\phi)^{4}+2 \sec (\phi)^{3}\left(g \lambda_{\psi} \eta_{\text {prop }} \rho S \cos (\phi)-4 K W^{2} \sin (\phi)\right) \tan (\phi)}{\eta_{\text {prop }} \rho S V}$, $\beta=-\frac{g \lambda_{\psi} \sec (\phi)^{2}}{V^{2}}+\frac{4 K W^{2} \sec (\phi)^{2} \tan (\phi)}{\eta_{\text {prop }} \rho S V^{2}}$, $\gamma=\frac{8 K W^{2} \sec (\phi)^{2}}{\eta_{\text {prop }} \rho S V^{3}}-\frac{3 \rho S V\left(C_{D_{o}}+\frac{4 K W^{2} \sec (\phi)^{2}}{\rho^{2} S^{2} V^{4}}\right)}{\eta_{\text {prop }}}+\frac{2 g \lambda_{\psi} \tan (\phi)}{V^{3}}$.

In order to check that the second order necessary condition is satisfied, we show that the left hand side of (68) is negative definite. This holds by Sylvester's Criterion, if, and only if, the determinant of the first nested principal minor of (69) is negative and the determinant of (69) is positive.

We will begin with the first nested principal minor as

$\frac{\eta_{\text {prop }} \eta_{\text {sol }} \rho P_{s d} S^{2} V(-(\cos (\phi) \sin (e))+\cos (e) \sin (a-\psi) \sin (\phi))}{\eta_{\text {prop }} \rho S V}+\frac{-4 K W^{2} \sec (\phi)^{4}+2 \sec (\phi)^{3}\left(g \lambda_{\psi} \eta_{\text {prop }} \rho S \cos (\phi)-4 K W^{2} \sin (\phi)\right) \tan (\phi)}{\eta_{\text {prop }} \rho S V}$.

By simplifying and assuming $\phi=0$ according to Proposition 1, we obtain,

$$
-4 K W^{2}-\eta_{\text {prop }} \eta_{\text {sol }} \rho P_{s d} S^{2} V \sin (e)
$$

which is always negative, and the first criterion is satisfied.

We must next examine the determinant of (69) which, if $\phi=0$, reduces to

$$
\begin{aligned}
& \left(-g^{2} \lambda_{\psi} \eta_{\text {prop }}^{2} \rho^{2} S^{2}+16 K^{2} W^{4}+12 C_{D_{o}} K \rho^{2} S^{2} V^{4} W^{2}+3 C_{D_{o}} \eta_{\text {prop }} \eta_{\text {sol }} \rho^{2} P_{s d} S^{4} V^{5} \sin (e)\right. \\
& \left.+4 K \eta_{\text {prop }} \eta_{\text {sol }} \rho P_{s d} S^{2} V W^{2} \sin (e)\right) / V^{4}
\end{aligned}
$$


In (72), we substitute expression (44) for $\lambda_{\psi}$ to obtain

$$
(A+D)(3 C+D)-B^{2},
$$

where

$$
\begin{aligned}
& A=V P_{s d} \rho S^{2} \eta_{\text {prop }} \eta_{\text {sol }} \sin (e), \\
& B=V P_{s d} \rho S^{2} \eta_{\text {prop }} \eta_{\text {sol }} \cos (e) \sin (a-\psi), \\
& C=C_{D_{o}} \rho^{2} S^{2} V^{4}, \\
& D=4 K W^{2} .
\end{aligned}
$$

If $P_{s d}$ is small enough, then expression (73) is positive, which completes the proof.

\section{References}

${ }^{1}$ F. Irving, D. Morgan, "The Feasibility of an Aircraft Propelled by Solar Energy," AIAA, 1974

${ }^{2}$ D. Hall, C. Fortenbach, E. Dimiceli, R. Parks, "A preliminary Study of Solar Powered Aircraft and Associated Power Trains," NASA Contractor Report 3699, 1983

${ }^{3}$ W. Phillips, "Some Design Considerations for Solar-Powered Aircraft," NASA Technical Paper 1975

${ }^{4}$ P. MacCready, P. LissaMan, W. Morgan, J. Burke, "Sun Powered Aircraft Design," AIAA 1981

${ }^{5}$ J. Youngblood, T. Talay, "Solar-Powered Airplane Design for Long-Endurance, High-Altitude Flight," AIAA 1982

${ }^{6}$ R. Boucher, "History of Solar Flight," AIAA, 1984

${ }^{7}$ J. Youngblood, T. Talay, R. Pegg, "Design of Long-Endurance Unmanned Airplanes Incorporating Solar and Fuel Cell Propulsion," AIAA, 1984

${ }^{8}$ P. Stella, D. Flood, "Photovoltaic Options for Solar Electric Propulsion," AIAA 1990

${ }^{9}$ N. Colella, G. Wenneker, "Pathfinder and the Development of Solar Rechargeable Aircraft," Energy and Technology Review, 1994 1995

${ }^{10}$ S. Brandt, F. Gilliam, "Design Analysis Methodology for Solar-Powered Aircraft," Journal of Aircraft vol. 32, pp 703-709,

${ }^{11}$ K. Reinhardt, T. Lamp, J. Geis, A. Colozza, "Solar-Powered Unmanned Aerial Vehicles," IEEE, 1996

${ }^{12}$ O. Trifu, G. Savu, "Unmanned Solar-Powered Aerial Surveyor Configured with an Aerodynamic Optimization Procedure," AIAA, 1997

${ }^{13}$ A. Colozza, D. Scheiman, D. Brinker, "GaAs/Ge Solar Powered Aircraft," NASA/TM, 1998

${ }^{14}$ K. Flittie, B. Curtin, "Pathfinder Solar-Powered Aircraft Flight Performance," AIAA 1998

${ }^{15}$ P. Berry, "The Sunriser - A Design Study in Solar Powered Flight," AIAA, SAE, 2000

${ }^{16}$ C. Wilson, J. Nutbean, I Bond, "Aerodynamice and Structural Design of a Solar-Powered Micro Unmanned Air Vehicle," IMechE, 2000

${ }^{17}$ G. Frulla, "Preliminary Reliability Design of a Solar-Powered High-Altitude Very Long Endurance Unmanned Air Vehicle," IMechE, 2002

${ }^{18}$ C. Patel, H. Arya, K. Sudhakar, "Design, Build, and Fly a Solar Powered Aircraft," Indian Institute of Technology, 2002

${ }^{19}$ C. Roberts, M. Vaughan, W. Bownman, "Development of a Solar Powered Micro Air Vehicle," AIAA, 2002

${ }^{20}$ M. Dornheim, "Get Me Through the Night Batteries are now challenging fuel cells to assist long-endurance solar-powered aircraft," Aviation week and Space Technology, vol. 159, iss. 11, pp 66, Sept 15, 2003

${ }^{21}$ A. Colozza, "Solid State Aircraft," NASA/DoD Conference on Evolvable Hardware, 2004

${ }^{22}$ A. Noth, W. Engel, R. Siegwart, "Design of an Ultra-Lightweight Autonomous Solar Airplane for Continuous Flight," Autonomous Systems Lab, EPFL, 2004

${ }^{23}$ C. Theodore, M. Tischler, J. Colbourne, "Rapid Frequency-Domain Modeling Methods for Unmanned Aerial Vehicle Flight Control Applications," Journal of Aircraft, 2004

${ }^{24}$ M. Dornheim, "Perpetual Motion," Aviation Week and Space Technology, 2005

${ }^{25}$ G. Romeo, G. Frulla, E. Cestino, F. Borello, "SHAMPO: Solar HALE Aircraft for Multi Payload and Operations," AIDAA, 2005

${ }^{26}$ N. Baldock, M. Mokhtarzadey-Dehghan, "A Study of Solar-Powered, High-Altitude, Unmanned Aerial Vehicles," Aircraft Engineering and Aerospace Technology: An International Journal, vol. 78, pp. 187-193, 2006

${ }^{27}$ M. Curry, "NASA Fact Sheet: Solar-Power Research and Dryden," Available online at: www.nasa.gov/centers/dryden/news/FactSheets/FS-054-DFRC.html, 2006

${ }^{28}$ D. Hall, D. Watson, R. Tuttle, S. Hall, "Mission Analysis of Solar Powered Aircraft," NASA Contractor Report 172583, 1985

${ }^{29}$ A. Colozza, "Effect of Power System Technology and Mission Requirements on High Altitude Long Endurance Aircraft," NASA Contractor Report 194455, 1994

${ }^{30}$ A. Colozza, "Effect of Date and Location on Maximum Achievable Altitude for a Solar Powered Aircraft," NASA Contractor Report 202326, 1997

${ }^{31}$ E. Teets, C. Donohue, P. Wright, "Meteorological Support of the Helios World Record High Altitude Flight to 96,863 Feet," NASA Technical Memorandum, 2002 
${ }^{32}$ A. Colozza, "Effect of Power System Technology and Mission Requirements on High Altitude Long Endurance Aircraft," NASA, 1994

${ }^{33}$ J. Gundlach, "Unmanned Solar-Powered Hybrid Airships for Mars Exploration," AIAA, 1999

${ }^{34}$ S. Smith, A. Hahn, W. Johnson, D. Kinney, J. Pollitt, J. Reuther, "The Design of the Canyon Flyer, An Airplane for Mars Exploration," AIAA, 2000

${ }^{35}$ A. Colozza, "Solar Powered Flight on Venus," NASA Contractor Report 213052, 2004

${ }^{36}$ A. Noth, S. Bouabdallah, S. Michaud, R. Siegwart, W. Engel, "Sky-Sailor: Design of an Autonomous Solar Powered Martian Airplane,", Autonomous Systems Lab, Swiss Federal Institute of Technology, 2004

${ }^{37}$ M. Mondin, F. Dovis, P. Mulassano, "On the Use of HALE Platforms as GSM Base Stations," IEEE Personal Communications, 2001

${ }^{38}$ T. Tozer, D. Grace, "High-Altitude Platforms for Wireless Communications," Electronics and Communication Engineering Journal, 2001

${ }^{39}$ T. Tozer, D. Grace, "HeliNet - The European Solar-Powered HAP Project," Communications Research Group, University of York, 2001 2002

${ }^{40}$ G. Romeo, G. Frulla, "HELIPLAT: Aerodynamic and Structural Analysis of HAVE Solar Powered Platform," AIAA,

${ }^{41}$ M. Oodo, H. Tsuji, R. Miura, M. Maruyama, M. Suzuki, "Experiment of IMT-2000 Using Stratospheric-Flying SolarPowered Aircraft," IEEE, 2003

42 J. Wise, "Bertrand Piccard's Solar-Powered Flight Around the World," Popular Mechanics, Sept. 2005

${ }^{43}$ W. Brown, "The History of Power Transmission by Radio Waves," IEEE, 1984

${ }^{44}$ D. Chichka, J. Speyer, "Solar-Powered, Formation-Enhanced Aerial Vehicle Systems for Sustained Endurance," Proceedings of the American Control Conference, 1998

${ }^{45}$ A. Colozza, "Convective Array Cooling for a Solar Powered Aircraft," NASA Contractor Report 212084, 2003

${ }^{46}$ B. Bryson, Y. Ho, "Applied Optimal Control," Hemisphere Publishing Corporation, 1975

${ }^{47}$ J. Burrows, "Fuel Optimal Aircraft Trajectories with Fixed Arrival Times," AIAA, 1981

${ }^{48}$ P. Kabamba, S. Meerkov, F. Zeitz, "Optimal Path Planning for Unmanned Combat Aerial Vehicles to Defeat Radar Tracking," Journal of Guidance, Control, and Dynamics vol. 29, pp 279-288, 2006

${ }^{49}$ P. Hughes, "Spacecraft Attitude Dynamics," Dover Publications, 2004

${ }^{50}$ A. Klesh, "SolarBubbles Sponsorship Packet," SolarBubbles Student Team, 2007

${ }^{51}$ D. Burns, Y. Li, "Study of Aerodynamic Losses due to Solar Cells on Wing Surfaces," SolarBubbles Student Team, 2006

${ }^{52}$ W. Chen, "AE 590 Directed Study Solar-Powered Unmanned Air Vehicle," SolarBubbles Student Team, 2006

${ }^{53}$ J. Anderson, "Aircraft Performance and Design," McGraw-Hill Science/Engineering/Math, 1998

${ }^{54}$ A. Klesh, P. Kabamba, "Solar-Powered Unmanned Aerial Vehicles on Mars: Perpetual Endurance," International Astronautical Congress, Accepted, 2007

${ }^{55}$ C. Chen, "Linear System Theory and Design," Oxford University Press, 1984 